University of New Orleans

ScholarWorks@UNO

7-1-2011

\title{
Simplified design of thin-film polarizing beam splitter using embedded symmetric trilayer stack
}

R. M.A. Azzam

University of New Orleans, razzam@uno.edu

Follow this and additional works at: https://scholarworks.uno.edu/ee_facpubs

Part of the Electrical and Electronics Commons, and the Optics Commons

\section{Recommended Citation}

R. M. A. Azzam, "Simplified design of thin-film polarizing beam splitter using embedded symmetric trilayer stack," Appl. Opt. 50, 3316-3320 (2011)

This Article is brought to you for free and open access by the Department of Electrical Engineering at ScholarWorks@UNO. It has been accepted for inclusion in Electrical Engineering Faculty Publications by an authorized administrator of ScholarWorks@UNO. For more information, please contact scholarworks@uno.edu. 


\title{
Simplified design of thin-film polarizing beam splitter using embedded symmetric trilayer stack
}

\author{
R. M. A. Azzam \\ Department of Electrical Engineering, University of New Orleans, \\ New Orleans, Louisiana 70148, USA (razzam@uno.edu) \\ Received 28 March 2011; accepted 6 May 2011; \\ posted 17 May 2011 (Doc. ID 144834); published 1 July 2011
}

\begin{abstract}
An analytically tractable design procedure is presented for a polarizing beam splitter (PBS) that uses frustrated total internal reflection and optical tunneling by a symmetric LHL trilayer thin-film stack embedded in a high-index prism. Considerable simplification arises when the refractive index of the highindex center layer $\mathrm{H}$ matches the refractive index of the prism and its thickness is quarter-wave. This leads to a cube design in which zero reflection for the $p$ polarization is achieved at a $45^{\circ}$ angle of incidence independent of the thicknesses of the identical symmetric low-index tunnel layers L and L. Arbitrarily high reflectance for the $s$ polarization is obtained at subwavelength thicknesses of the tunnel layers. This is illustrated by an IR Si-cube PBS that uses an embedded ZnS-Si-ZnS trilayer stack. (c) 2011 Optical Society of America
\end{abstract}

OCIS codes: $\quad 230.1360,230.5440,240.0310,260.5430,310.6860$.

\section{Introduction}

Thin-film polarizing beam splitters (PBSs) are versatile optical elements that use destructive interference of light for one linear polarization ( $p$ or $s$ ) and nearly full constructive interference for the orthogonal polarization in a multilayer stack at oblique incidence [1-10].

This paper follows up on previous work by Azzam and Perla $[11,12]$ that dealt with the polarizing properties of a symmetric LHL trilayer stack (that consists of a high-index center-layer $\mathrm{H}$ of refractive index $n_{2}$ and thickness $d_{2}$ sandwiched between two identical low-index tunnel layers $\mathrm{L}$ of refractive index $n_{1}$ and thickness $d_{1}$ ), which is embedded in a prism of high refractive index $n_{0}>n_{1}$, Fig. 1. All media are considered to be transparent and optically isotropic and are separated by parallel-plane boundaries. Light is incident from medium 0 at an angle $\phi_{0}>$ the critical angle $\phi_{c 01}=\arcsin \left(n_{1} / n_{0}\right)$ of the 01 interface, so that frustrated total internal reflection (FTIR) and optical tunneling through the trilayer stack take place. The optical field is evanescent in

0003-6935/11/193316-05\$15.00/0

(ㄷ) 2011 Optical Society of America the low-index tunnel layers and is propagating in the prism and center layer. In Sections 2 and 3 we consider an important and analytically träctable special case in which the refractive index of the center layer is deliberately matched to that of the prism, i.e., $n_{2}=n_{0}$. This simple design employs only two optical materials and depends on fewer design parameters, namely only one index ratio $N=n_{0} / n_{1}$, angle of incidence $\phi_{0}$, and film thicknesses $d_{1}, d_{2}$. A novel PBS cube $\left(\phi_{0}=45^{\circ}\right)$ is introduced in Section 4 that uses index ratio $N=(\sqrt{2}+1)^{1 / 2}=1.55377$ and center layer of quarter-wave optical thickness. This PBS, which uses tunnel layers of subwavelength thicknesses, fully transmits the $p$ polarization and nearly totally reflects the $s$ polarization. Section $\underline{5}$ provides a brief summary of this paper.

\section{Constraint on Film Thicknesses for Zero Reflection of the $p$ or $s$ Polarization}

In [11] it was shown that the condition of zero reflection of the $\nu$ polarization $(\nu=p, s)$ by an embedded symmetric trilayer stack can be put in the form

$$
X_{2}=\frac{\ell+m X_{1}-n X_{1}^{2}}{-n+m X_{1}+\ell X_{1}^{2}} .
$$




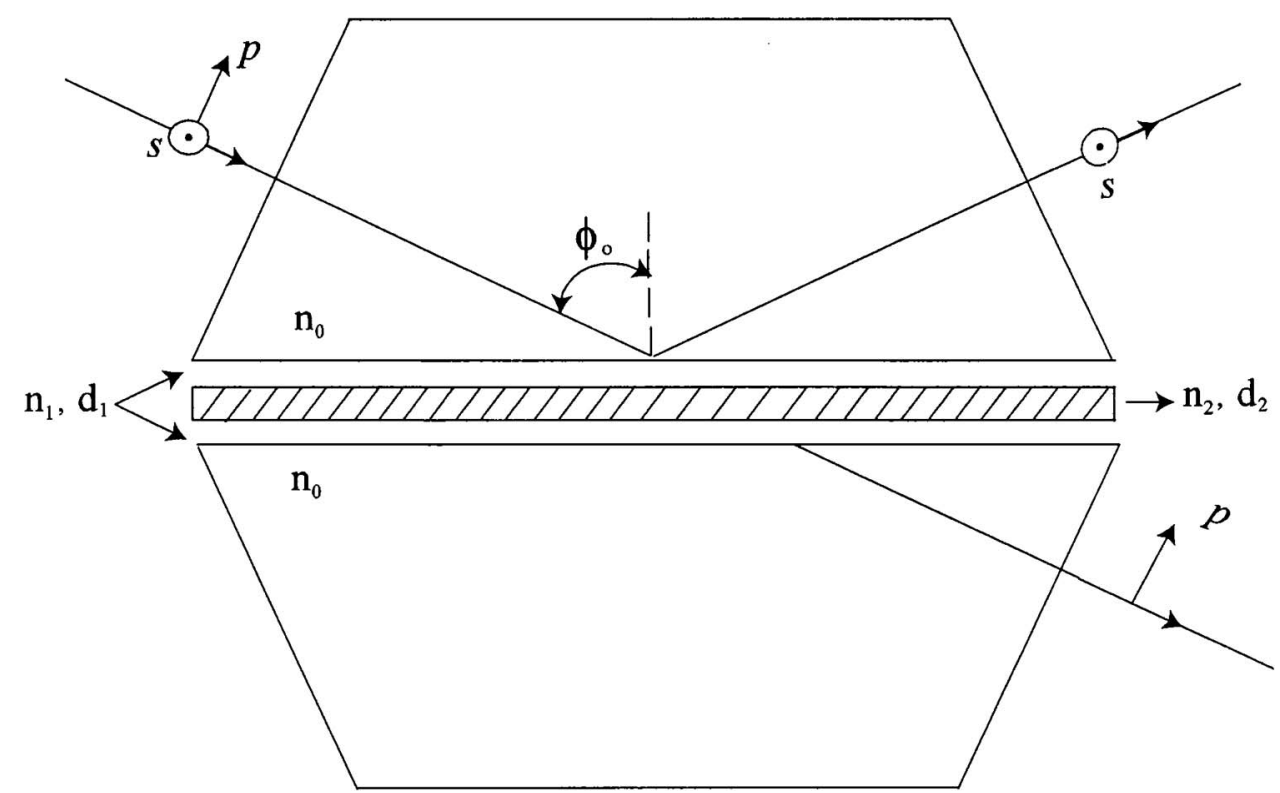

Fig. 1. Embedded symmetric trilayer stack as a PBS operating under conditions of FTIR. $p$ and $s$ are the linear polarizations parallel and perpendicular to the plane of incidence, respectively, and $\phi_{0}$ is the angle of incidence.

When index matching $\left(n_{2}=n_{0}\right)$ is satisfied, $\ell, m$, and $n$ in Eq. (1) are determined by only one Fresnel reflection coefficient $r_{01 \nu}$ at the 01 interface:

$$
\ell=r_{01 \nu}, \quad m=-r_{01 \nu}\left(1+r_{01 \nu}^{2}\right), \quad n=-r_{01 \nu}^{3} .
$$

Upon substitution of $\ell, m$, and $n$ from Eqs. (2) in Eq. (1), the condition of zero reflection of the $\nu$ polarization reduces to

$$
X_{2}=\frac{1-r_{01 \nu}^{2} X_{1}}{r_{01 \nu}^{2}-X_{1}}
$$

In Eqs. (1) and (3) $X_{1}$ and $X_{2}$ are exponential functions of film thicknesses given by

$$
X_{i}=\exp \left[-j 4 \pi\left(n_{i} d_{i} / \lambda\right) \cos \phi_{i}\right], \quad i=1,2 .
$$

In Eq. (4), $\phi_{i}$ is the angle of refraction in the $i$ th layer, and $\lambda$ is the vacuum wavelength of light.

Since FTIR takes place at the 01 interface at $\phi_{0}$, the light field is evanescent in the low-index tunnel layers, $\cos \phi_{1}$ is pure imaginary, and $X_{1}$ is pure real in the range $0 \leq X_{1} \leq 1$. Also, because $n_{2}=n_{0}$, the angle of refraction in the high-index center layer $\phi_{2}=\phi_{0}$ and $X_{2}$ is a pure phase factor, i.e., $\left|X_{2}\right|=1$.

To further clarify the thickness constraint for zero reflection of the $\nu$ polarization, we substitute

$$
r_{01 \nu}=\exp (j \delta), \quad X_{2}=\exp \left(-j \theta_{2}\right),
$$

in Eq. (3) and solve for $\theta_{2}$ in terms of $\delta, X_{1}$ :

$$
\theta_{2}=2 \delta+2 \arg \left[1-X_{1} \exp (-j 2 \delta)\right] .
$$

Equation (6) is significant in that it is equally valid for the $p$ and $s$ polarizations (for simplicity the subscript $\nu$ is dropped from $\delta$ ) and depends only impli- citly on the index ratio $N$ and angle of incidence $\phi_{0}$ via the 01-interface reflection phase shift $\delta$.

From the round-trip phase thickness $\theta_{2}$ of the high-index center layer, the corresponding metric thickness $d_{2}$ is determined by

$$
d_{2} / \lambda=\left[\left(4 \pi n_{0}\right)^{-1} \sec \phi_{0}\right] \theta_{2} .
$$

Likewise, the metric thickness of the low-index tunnel layers is obtained from $X_{1}$ by

$$
d_{1} / \lambda=\frac{-\ln X_{1}}{\left(4 \pi n_{1}\right)\left(N^{2}-\sin ^{2} \phi_{0}\right)^{1 / 2}} .
$$

Figure 2 shows a family of curves of the normalized center-layer phase thickness $\theta_{2} / \pi$ as a function of the tunnel-layer thickness parameter $X_{1}$ in the range $0 \leq$ $X_{1} \leq 1$ as calculated from Eq. (6) for discrete values of the interface reflection phase shift $\delta=q \pi / 8, q=$ $0,1,2, \ldots, 8$ as a parameter. Limiting cases are identified from Eq. (ㅁ) and Fig. $\underline{2}$ as follows.

1. When $\delta=0, \theta_{2}=0$; and when $\delta=\pi, \theta_{2}=2 \pi$ independent of $X_{1}$. These limiting values of $\delta$ occur at the critical angle and grazing incidence [13], respectively, and are of little or no practical interest.

2 . In the limit as $X_{1} \rightarrow 0$, i.e., for tunnel-layer thickness of the order of a wavelength or greater, $\theta_{2} \rightarrow 2 \delta$. Such a condition is desirable for achieving high reflectance of the unextinguished orthogonal polarization as discussed in Section 3 .

3 . When $\delta=\pi / 2$ (01-interface reflection phase shift is quarter-wave), $\theta_{2}=\pi$ (center layer is of quarter-wave optical thickness at oblique incidence) independent of $X_{1}$. This interesting special case provides the basis of the unique PBS cube design described in Section $\underline{4}$. 


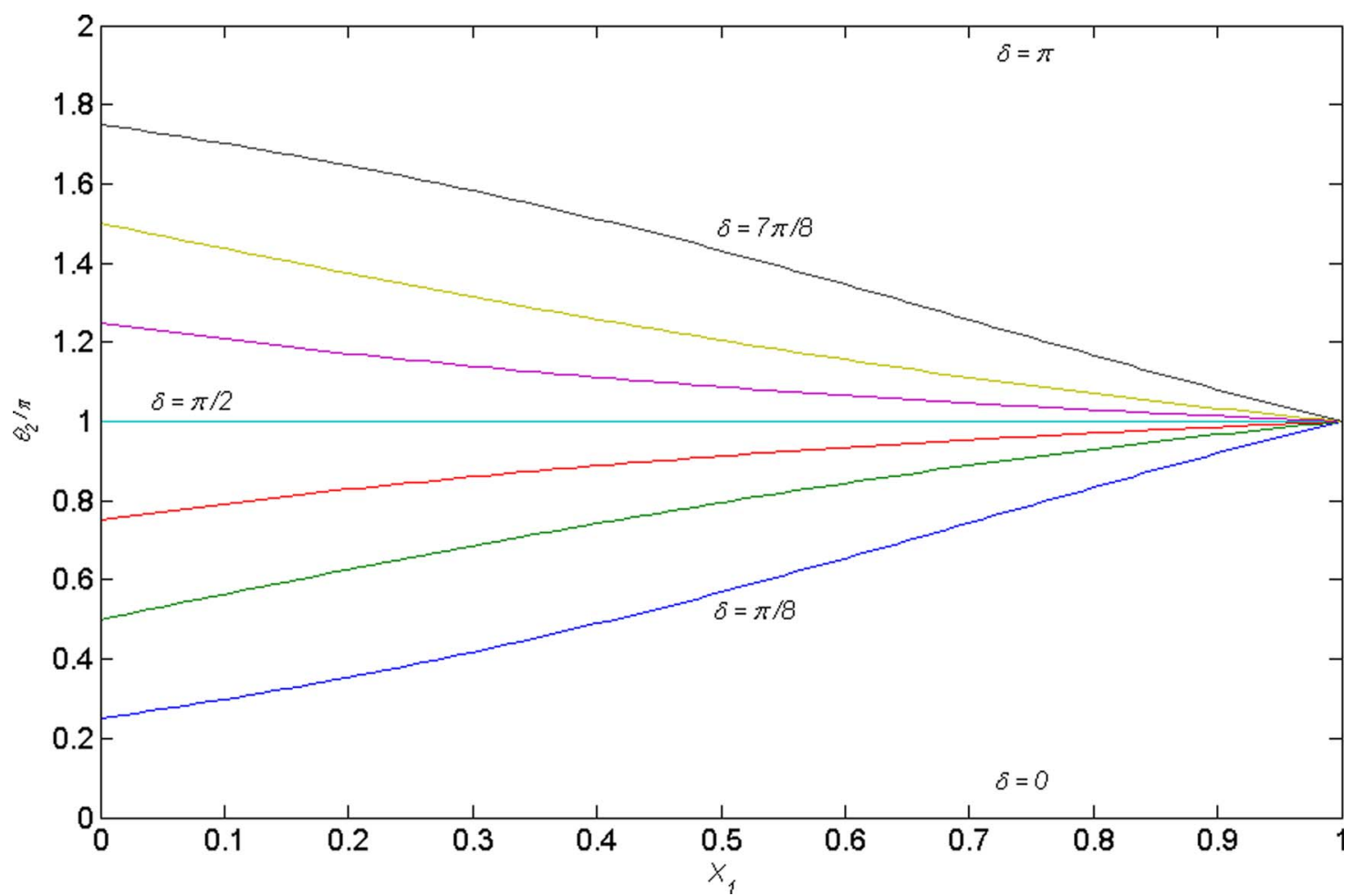

Fig. 2. (Color online) Normalized center-layer phase thickness $\theta_{2} / \pi$ is plotted as a function of the tunnel-layer thickness parameter $X_{1}$ using Eq. (6) at discrete values of the 01-interface reflection phase shift $\delta=q \pi / 8, q=0,1,2, \ldots, 8$ such that zero reflection is achieved for the $p$ or $s$ polarization.

\section{Embedded Symmetric Trilayer with Index-Matched Quarter-Wave-Thick Center Layer: Reflectance of the Orthogonal Polarization}

For an embedded symmetric trilayer stack with index-matched quarter-wave center layer (i.e., $n_{2}=$ $n_{0}, \theta_{2}=\pi, X_{2}=-1$ ) the complex-amplitude reflection coefficient for the orthogonal polarization $(o)$ is given by

$$
R_{o}=\frac{\left(1-X_{1}\right)^{2} \exp \left(j \delta_{o}\right)}{1-2 X_{1} \sec \delta_{o} \exp \left(j \delta_{o}\right)+X_{1}^{2} \exp \left(j 2 \delta_{o}\right)} .
$$

In Eq. (9) $\delta_{o}$ is the 01-interface reflection phase shift for the orthogonal polarization. The associated intensity reflectance is obtained from Eq. (9) as

$$
\left|R_{o}\right|^{2}=\frac{\left(1-X_{1}\right)^{4}}{\left(1-X_{1}\right)^{4}+4\left(\sec \delta_{o}-\cos \delta_{o}\right)^{2} X_{1}^{2}} .
$$

Figure 3 shows a family of curves of $\left|R_{o}\right|^{2}$ as a function of $\bar{X}_{1}$ in the range $0 \leq X_{1} \leq 0.1$ calculated from Eq. (10) at selected values of $\delta_{o}=q \pi / 8, q=$ $0,1,2, \overline{3}, 5,6,7,8$. In Fig. $3 \delta_{o}=\pi / 2$ is excluded as it renders $\left|R_{o}\right|^{2}=0$, which is not physically acceptable. Furthermore, $\delta_{o}=0, \pi$ correspond to total reflection, $\left|R_{o}\right|^{2}=1$, at the critical angle and grazing incidence, respectively, and are not of interest.

\section{PBS Cube Using Embedded Symmetric Trilayer with Index-Matched, Quarter-Wave-Thick Center Layer}

The $p$ polarization can be totally suppressed in reflection (hence is fully transmitted) by a symmetric tri- layer stack with index-matched center layer $n_{2}=n_{0}$ that is embedded in a cube prism of refractive index $n_{0}$. The 01-interface reflection phase shift $\delta_{p}=\pi / 2$ is achieved at $\phi_{0}=45^{\circ}$ if the index ratio $N$ is selected such that [13]

$$
\sin ^{2} \phi_{0}=1 / 2=\left(N^{2}+1\right) /\left(N^{4}+1\right) .
$$

Equation (11) yields

$$
N=n_{0} / n_{1}=(\sqrt{2}+1)^{1 / 2}=1.55377 .
$$

It is interesting to note that $N=1.55377$ causes maximum separation between the critical angle and the angle at which $\delta_{p}-\delta_{s}$ is maximum [13].

Table 1 lists some prism and film refractive indices that satisfy Eq. (12). For a given prism index $n_{0}$, the refractive index $\overline{n_{1}}$ of the tunnel layers is calculated from Eq. (12) and rounded to three decimal places.

As already noted in Section 2, zero reflection for the $p$ polarization is achieved when $\delta_{p}=\pi / 2$ and $\theta_{2}=\pi$ (center layer of quarter-wave optical thickness) independent of the thickness of the symmetric tunnel layers.

As a specific design, consider an IR-transparent $\mathrm{ZnS}-\mathrm{Si}-\mathrm{ZnS}$ trilayer that is embedded in a Si cubical prism with refractive indices $n_{0}=n_{2}=3.4, n_{1}=$ 2.188. Substitution of $n_{0}=3.4, \phi_{0}=45^{\circ}, \theta_{2}=\pi$ in Eq. (7) gives the required metric thickness of the center layer 


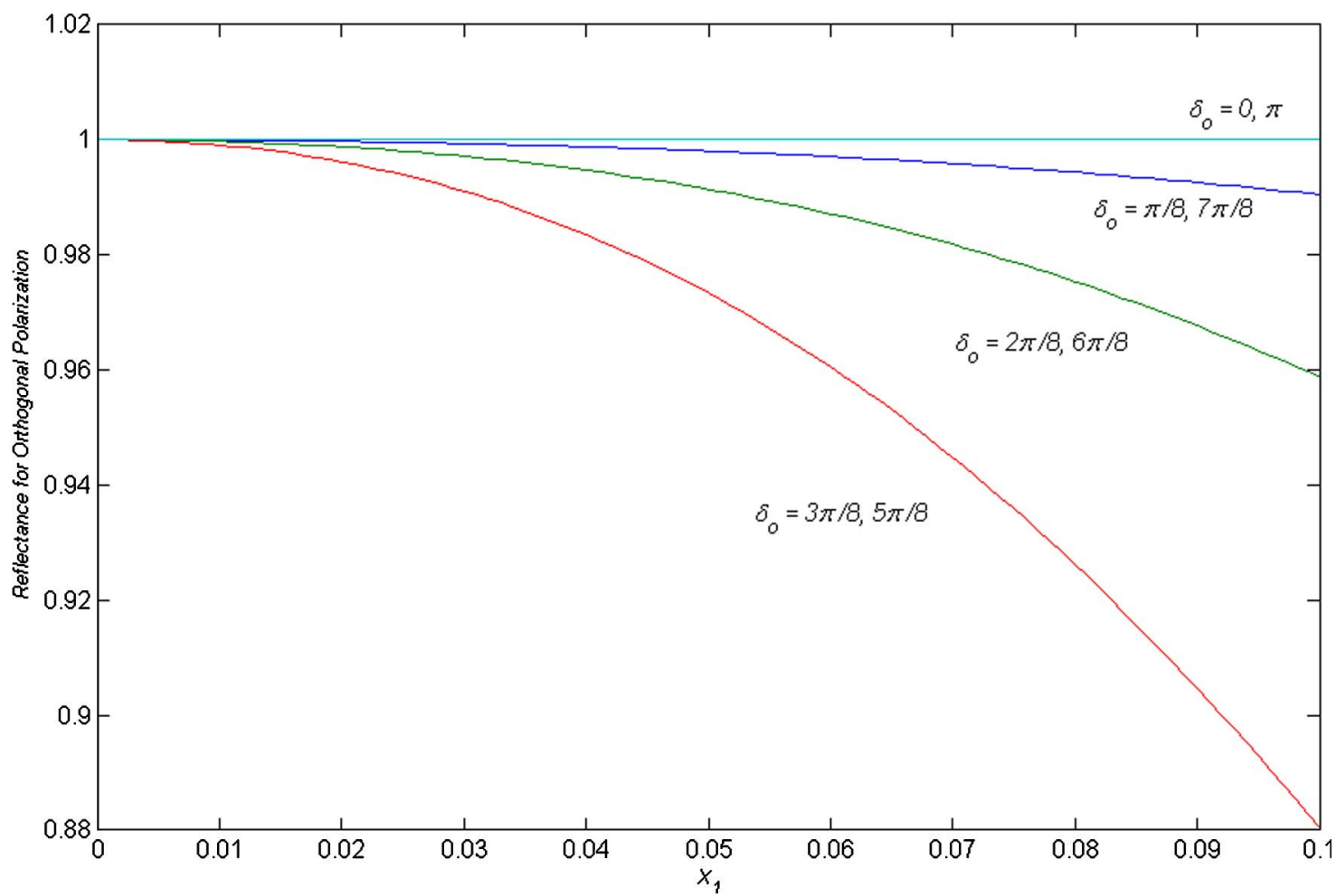

Fig. 3. (Color online) Intensity reflectance for the orthogonal polarization $\left|R_{o}\right|^{2}$ is plotted as a function of $X_{1}$ using Eq. (10) at selected values of $\delta_{o}=q \pi / 8, q=0,1,2,3,5,6,7,8$.

$$
d_{2}=0.10399 \lambda
$$

To calculate the reflectance for the orthogonal $(o \equiv s)$ polarization, Abelès's condition [14] at $\phi_{0}=45^{\circ}$ is used to obtain

$$
\delta_{o}=\delta_{s}=\delta_{p} / 2=\pi / 4
$$

Substitution of $\delta_{o}=\pi / 4$ in Eq. (10) gives

$$
\left|R_{s}\right|^{2}=\frac{\left(1-X_{1}\right)^{4}}{\left(1-X_{1}\right)^{4}+2 X_{1}^{2}} .
$$

From Eq. (16) it is apparent that the reflectance for the $s$ polarization can be made as high as possible, without affecting the condition of zero reflection for the $p$ polarization, by increasing the thickness of the tunnel layers to make $X_{1}$ as small as one wishes. For example, if $X_{1}=0.001$ is substituted in Eq. (16), $\left|R_{s}\right|^{2}=0.999998$ is obtained. The metric thickness of the tunnel layers is calculated by substituting $X_{1}=0.001, N=(\sqrt{2}+1)^{1 / 2}, n_{1}=2.188, \quad \phi_{0}=45^{\circ}$ in Eq. (8); this gives

$$
d_{1}=0.18159 \lambda \text {. }
$$

From Eqs. (16) and (17) the total thickness of the trilayer stack,

$$
d_{\text {tot }}=d_{2}+2 d_{1}=0.46717 \lambda .
$$

For a $\mathrm{CO}_{2}$-laser wavelength $\lambda=10.6 \mu \mathrm{m}, \quad d_{\text {tot }}=$ $4.952 \mu \mathrm{m}$.

The design presented in this section is intended for operation at a single (e.g., laser) wavelength or within a narrow bandwidth centered at a given $\lambda$. This is obvious from Eqs. (13), (17), and (18), which show that the required layer thicknesses are specific fractions of the wavelength of incident light. When a collimated laser source is used, the limited field of view of the PBS is not a significant concern. As is well known, the design of broadband and wide-field-ofview PBS requires the use of a large number of thin films (see, e.g., $[\underline{8}, \underline{9}, \underline{15}]$ ).

\section{Summary}

Considerable simplification is achieved in the design of PBSs that employ symmetric LHL trilayers embedded in a high-index prism when the refractive indices of the high-index center layer and the prism are matched. The $p$ polarization is suppressed in reflection independent of the thicknesses of the tunnel

Table 1. Selected Prism and Film Refractive Indices That Satisfy Eq. (12) ${ }^{a}$

\begin{tabular}{lccccc}
\hline$n_{0}$ (prism) & 1.55377 (glass) & $2.2(\mathrm{ZnS})$ & $2.35(\mathrm{ZnS})$ & $3.4(\mathrm{Si})$ & $4(\mathrm{Ge})$ \\
$n_{1}$ (thin film) & 1 & 1.416 & 1.512 & 2.188 & 2.574 \\
\hline
\end{tabular}

\footnotetext{
${ }^{a}$ Refractive indices of $\mathrm{ZnS}$ listed in the third and fourth columns are appropriate to the IR and visible spectrum, respectively.
} 
layers at a $45^{\circ}$ angle of incidence (PBS cube) by using an index-matched center layer of quarter-wave thickness. High reflectance of the $s$ polarization is obtained by suitable choice of the thickness of the tunnel layers. One such Si-cube PBS is described that uses an embedded $\mathrm{ZnS}-\mathrm{Si}-\mathrm{ZnS}$ stack in the IR.

I am pleased to thank Ryan Adams for assistance with the figures.

\section{References}

1. S. M. MacNeille, "Beam splitter," U. S. patent 2,403,731 (9 July 1946).

2. M. Banning, "Practical methods of making and using multilayer filters," J. Opt. Soc. Am. 37, 792-795 (1947).

3. R. P. Netterfield, "Practical thin-film polarizing beam splitters," J. Mod. Opt. 24, 69-79 (1977).

4. D. Lees and P. Baumeister, "Versatile frustrated-totalreflection polarizer for the infrared," Opt. Lett. 4, 66-67 (1979).

5. R. M. A. Azzam, "Polarizing beam splitters for infrared and millimeter waves using single-layer-coated dielectric slab or unbacked films," Appl. Opt. 25, 4225-4227 (1986).

6. M. Gilo and K. Rabinovitch, "Design parameters of thin-film cubic-type polarizers for high power lasers," Appl. Opt. 26, 2518-2521 (1987).
7. J. Mouchart, J. Begel, and E. Duda, "Modified MacNeille cube polarizer for a wide angular field," Appl. Opt. 28, 2847-2853 (1989).

8. L. Li and J. A. Dobrowolski, "Visible broadband, wide-angle, thin-film multilayer polarizing beam splitter," Appl. Opt. 35, 2221-2225 (1996).

9. L. Li and J. A. Dobrowolski, "High-performance thin film polarizing beam splitter operating at angles greater than the critical angle," Appl. Opt. 39, 2754-2771 (2000).

10. B. V. Blanckenhagen, "Practical layer design for polarizing beam-splitter cubes," Appl. Opt. 45, 1539-1543 (2006).

11. R. M. A. Azzam and S. R. Perla, "Polarizing properties of embedded symmetric trilayer stacks under conditions of frustrated total internal reflection," Appl. Opt. 45, 1650-1656 (2006).

12. R. M. A. Azzam and S. R. Perla, "Errata," Appl. Opt. 46, 431-433 (2007).

13. R. M. A. Azzam, "Phase shifts that accompany total internal reflection at a dielectric-dielectric interface," J. Opt. Soc. Am. A 21, 1559-1563 (2004).

14. F. Abelès, "Un théoreme relatif à la réflexion métallique," C. R. Hebd. Seances Acad. Sci. 230, 1942-1943 (1950).

15. S. R. Perla and R. M. A. Azzam, "Wide-angle, highextinction-ratio, infrared polarizing beam splitters using frustrated total internal reflection by an embedded centro-symmetric multilayer," Appl. Opt. 46, 4604-4612 (2007). 